INPLASY

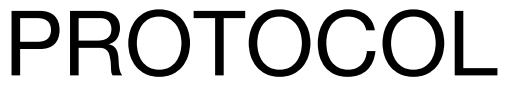

To cite: Zhang. Exercise intervention for sleep disorders after stroke: a protocol for systematic review and metaanalysis. Inplasy protocol 202130106. doi:

10.37766/inplasy2021.3.0106

Received: 29 March 2021

Published: 29 March 2021

Corresponding author:

Qin Zhang

1615793515@qq.com

Author Affiliation:

Emergency Department, West

China Hospital

Support: None.

Review Stage at time of this submission: The review has not yet started.

Conflicts of interest:

None declared.

\section{Exercise intervention for sleep disorders after stroke: a protocol for systematic review and meta-analysis}

\author{
Zhang, Q1.
}

Review question / Objective: The purpose of this systematic review is to evaluate the effectiveness and safety of exercise in improving sleep disorders after stroke, in order to obtain scientific exercise programs.

Condition being studied: After stroke, sleep disorder (PSSD) is the most common complication of stroke. After stroke, The brain cells of the body are damaged to varying degrees, To cause the release of toxic substances, These toxic substances can negatively affect the reticular activation of the brain, Damage to the sleep - arousal system, Which leads to sleep disorders, induce sleep disorders. If the patient's sleep quality does not improve for a long time, Highly prone to negative emotions such as anxiety, depression, Compounding the psychological and physical suffering of the patient, Causes damage to his motor and neurological functions, unfavorable for prognostic. Such and other reports showed that the incidence of sleep disorders after stroke was as high as 44.20. Sleep disorders have serious negative effects on the recovery of neurological function and the quality of life of patients with stroke, Also increases the risk of stroke recurrence, exacerbating neurological deficits in stroke patients, Even increases mortality and cognitive impairment.

INPLASY registration number: This protocol was registered with the International Platform of Registered Systematic Review and Meta-Analysis Protocols (INPLASY) on 29 March 2021 and was last updated on 29 March 2021 (registration number INPLASY202130106).

\section{INTRODUCTION}

Review question / Objective: The purpose of this systematic review is to evaluate the effectiveness and safety of exercise in improving sleep disorders after stroke, in order to obtain scientific exercise programs.

Condition being studied: After stroke, sleep disorder (PSSD) is the most common complication of stroke. After stroke, The 
brain cells of the body are damaged to varying degrees, To cause the release of toxic substances, These toxic substances can negatively affect the reticular activation of the brain, Damage to the sleep - arousal system, Which leads to sleep disorders, induce sleep disorders. If the patient's sleep quality does not improve for a long time, Highly prone to negative emotions such as anxiety, depression, Compounding the psychological and physical suffering of the patient, Causes damage to his motor and neurological functions, unfavorable for prognostic. Such and other reports showed that the incidence of sleep disorders after stroke was as high as 44.20. Sleep disorders have serious negative effects on the recovery of neurological function and the quality of life of patients with stroke, Also increases the risk of stroke recurrence, exacerbating neurological deficits in stroke patients, Even increases mortality and cognitive impairment.

\section{METHODS}

Participant or population: exercise in improving sleep disorders in stroke patients.

Intervention: Our systematic review and meta-analysis will be based on the recognition of the application of various forms of exercise intervention in the experimental group, including aerobic exercise, anaerobic exercise, flexion and extension exercise.

Comparator: Control group should did not receive exercise intervention.

Study designs to be included: Randomized controlled trials.

Eligibility criteria: Repeated published literature with poor quality assessment; randomised controlled trials of exercise combined with other therapies; literature without access to raw data will be excluded.

Main outcome(s): The main results of this study are that the effect of rehabilitation training for sleep disorders after stroke is mainly evaluated by the changes of the scores before and after the Pittsburgh Sleep quality Index scale.

Data management: Develop a unified data extraction table that includes authors, countries, regions, year of publication, sample size, inclusion criteria, patient characteristics (age and sex, body mass index (BMI), type of stroke, duration, lesion location, compliance with sleep disorders, and use of sleep disorders drugs), interventions (type, frequency, intensity, and time of exercise), place of intervention (hospital, community, home, and professional institution of exercise), time of follow-up, outcome indicators: score of the Pittsburgh Sleep Quality Index Scale, type and quantity of adverse events, cause, time and quantity of death. the process was performed independently by two researchers. if the main results data were not available, the study would be excluded; the results were reviewed by a third researcher. If the extraction information is lost or unclear, the author of the literature will be contacted by mail to obtain the data.

Quality assessment / Risk of bias analysis: The selected randomized controlled trial will use the Cochrane cooperation bias risk tool to evaluate quality of the included literature.

Strategy of data synthesis: Data analysis uses RevMan Manager 5.3 (Cochrane collaboration) and STATA 16.0 software to calculate mean deviations, standard deviations, confidence intervals, and $P$ values. For continuity variables, If the results are obtained with the same measuring tool, we will use weighted mean difference analysis; If the same variable uses a different measuring tool, we use standardized mean difference analysis.

Subgroup analysis: If the number of studies we've included is sufficient, the subgroup analysis will be used.

Sensitivity analysis: We will observe whether there are significant changes in the synthesis results by excluding each individual randomized controlled trial one 
by one. If change is considered unstable, vice versa.

Country(ies) involved: China.

Keywords: Sleep disorders after stroke, exercise.

Contributions of each author:

Author 1 - Qin Zhang.

Email: 1615793515@qq.com 